\title{
A Historical Perspective on Presentations of Hypertensive Acute Heart Failure
}

Chad E Darling ${ }^{1 *}$, Jiaoyuan Elisabeth Sun ${ }^{1}$, Jordan Goldberg' ${ }^{1}$ Peter Pang ${ }^{4}$, Christopher W Baugh ${ }^{5}$, Darleen Lessard ${ }^{2}$ and David D McManus $^{3}$

${ }^{1}$ Department of Emergency Medicine, University of Massachusetts Medical School, Worcester, MA, USA

${ }^{2}$ Department of Quantitative Health Sciences, University of Massachusetts Medical School, Worcester, MA, USA

${ }^{3}$ Department of Medicine, Division of Cardiology, University of Massachusetts Medical School, Worcester, MA, USA

${ }^{4}$ Department of Emergency Medicine, University of Indiana, Indianapolis, IN, USA

${ }^{5}$ Department of Emergency Medicine, Brigham and Women's Hospital, Boston, MA, USA

\begin{abstract}
Background: The initial systolic blood pressure (SBP) in patients presenting to the hospital with acute heart failure (AHF) informs prognosis, diagnosis, and guides initial treatment. However, over time AHF presentations with elevated SBP appear to have declined. The present study examined whether the frequency of AHF presentations with systolic hypertension (SBP $>160 \mathrm{mmHg}$ ) declined over a nearly two-decade time interval.

Methods: This study compares four historical, cross-sectional cohorts with AHF who were admitted to tertiary care medical centres in the North-eastern USA in 1995, 2000, 2006, and 2011-13. The main outcome was the proportion of AHF patients presenting with an initial SBP $>160 \mathrm{mmHg}$.

Results: 2,366 patients comprised the study sample. The average age was 77 years, $55 \%$ were female, $94 \%$ white, and $75 \%$ had prior heart failure. In $1995,34 \%$ of AHF patients presented with an initial SBP >160 mmHg compared to $20 \%$ in 2011-2013 ( $p<0.01$ ). Multivariate logistic regression demonstrated reduced odds of presenting with a SBP >160 mmHg in $2006(0.64,95 \% \mathrm{Cl} 0.42-0.96)$ and $2011-13(0.46,95 \% \mathrm{Cl} 0.28-0.74)$ compared with patients in 1995.

Conclusion: The proportion of patients with AHF and initial SBP $>160 \mathrm{mmHg}$ significantly declined over the study time period. There are several potential reasons for this observation and these findings highlight the need for ongoing surveillance of patients with AHF as changing clinical characteristics can impact early treatment decisions.
\end{abstract}

Keywords: Acute heart failure; Systolic blood pressure; Emergency department; Epidemiology

Abbreviations: AHF: Acute Heart Failure; SBP: Systolic Blood Pressure; ED: Emergency Department; WHFS: Worcester Heart Failure Study

\section{Introduction}

Worldwide, over 20 million people have chronic heart failure (HF) [1]. Patients with HF are prone to recurrent acute HF (AHF) exacerbations, and in the United States the majority of such patients are cared for in an emergency department (ED). Acute HF presentations account for nearly one million visits [2] and hospital admissions annually in the U.S. alone [3,4]. Efficiently diagnosing AHF and initiating appropriate therapies for patients with AHF can be challenging since multi-morbidity is common in patients with HF. However, several clinical parameters available at the time of hospital presentation, including history, exam, lab testing, xray, EKG, and point of care ultrasound, provide information that is useful in diagnosing the presence of AHF and guiding treatment [5]. Expert opinion has suggested that the initial, ED management of patients with AHF be based on the patient's clinical profile [6]. This is, in part, influenced by vital signs at the time of presentation and in particular presenting blood pressure (BP) $[7,8]$.

Historically the majority of patients with AHF present to the hospital with either elevated or normal blood pressure (BP) [7-9]. These initial $\mathrm{BP}$ values can be used to classify patients as having either "vascular failure," as defined by a systolic blood pressure (SBP) of $>160 \mathrm{mmHg}$, or "cardiac failure," as defined by a normal or low SBP (e.g., $<150 \mathrm{mmHg}$ ) $[9,10]$. Although far from an exact science, presenting blood pressure profile is related to type of $\mathrm{HF}$, with patients having preserved ejection fraction (HFpEF) more commonly presenting with an elevated SBP $[5,9]$. Since treatment decisions about AHF must frequently be made in the ED without echocardiographic data, SBP represents a potential initial guide for early AHF management.

Epidemiological studies suggest that the clinical characteristics, management, and prognosis of patients with HF have changed over the past several decades. Compared to prior years, contemporary patients presenting with AHF are older, have a greater comorbidity burden, and have increased short and longer-term survival [11]. Although the prognosis from HF has improved over time, rates of hospital admission for HF are not in decline, suggesting that AHF remains a common and costly condition. Between the 1990s and the mid-2000s it appeared that there was a decline in the number of patients presenting with AHF and elevated systolic hypertension on admission. We undertook the present study to examine whether there was indeed a historical change in the frequency of AHF presentations with systolic hypertension (SBP $>160$ $\mathrm{mmHg}$ ) on arrival to the ED in 4 historical, cross-sectional, cohorts between 1995 and 2013.

\section{Methods}

\section{Study population sources}

We derived our study population from data collected on patients

*Corresponding authors: Chad E Darling, Department of Emergency Medicine, University of Massachusetts Medical School, 55 Lake Avenue North Worcester MA, 01655, USA, Tel: 5084211464; E-mail: chad.darling@umassmed.edu

Received April 12, 2017; Accepted May 12, 2017; Published May 15, 2017

Citation: Darling CE, Sun JE, Goldberg J, Pang P, Baugh CW, et al. (2017) A Historical Perspective on Presentations of Hypertensive Acute Heart Failure. J Cardiovasc Dis Diagn 5: 275. doi: 10.4172/2329-9517.1000275

Copyright: (c 2017 Darling CE, et al. This is an open-access article distributed under the terms of the Creative Commons Attribution License, which permits unrestricted use, distribution, and reproduction in any medium, provided the original author and source are credited. 
seen in the EDs of 3 teaching hospitals in Worcester, MA (USA) and subsequently admitted to the hospital with a diagnosis of AHF. Two National Institute of Health sponsored research projects, conducted by members of the same research group, contributed to the database for the present study. The first study was the Worcester heart failure study (WHFS) which was a large, multi-center investigation that documented the clinical characteristics, treatments, and outcomes of adult patients admitted with AHF to all medical centers in central Massachusetts over multiple study years between 1995 and 2006 [11,12]. The second study was an ED-based extension of the WHFS using similar methodology and was conducted from 2011-2013. Both studies conform with the principles outlined in the Declaration of Helsinki and underwent full review by the Committee for the Protection of Human Subjects in Research at the University of Massachusetts Medical School. All study activities described below were approved, and all subjects either signed a written informed consent document prior to participation or were considered exempt.

\section{Additional Study Details}

\section{The Worcester heart failure study}

The WHFS collected data on adult patients admitted to multiple $(n=11)$ hospitals in central Massachusetts (MA) over several study years between 1995 and 2006 [11,12]. For the present study, we restricted the WHFS sample to patients admitted in 1995, 2000, and 2006 to three teaching hospitals in Worcester, MA. Patients in the WHFS were identified by trained study staff who performed a standardized review of the medical records of patients with primary and/or secondary International Classification of Diseases (9th Revision) diagnoses consistent with a possible admission for AHF. Patients who were discharged with the HF code of (428.XX) were used as the primary means for identifying cases of possible AHF. Subsequently only cases that met the Framingham criteria for HF were included in the final dataset [13]

\section{ED-based study}

The ED-based, observational, investigation began in September 2011, and enrolled patients over 2 years from same teaching hospitals included in the WHFS. Patients were identified and initially enrolled in the ED by trained study staff based on history, physical exam, physician impression, and lab values consistent with the occurrence of possible AHF. The HF diagnosis was confirmed (or refuted) by subsequent chart review based on whether the subject met Framingham criteria for AHF as in the WHFS.

\section{Inclusion and exclusion criteria}

Only adult ( $>18)$ patients admitted to one of the study teaching hospitals after being evaluated in the ED were included for both the WHFS and ED enrolled patients. As mentioned, our diagnostic criterion for AHF was the Framingham criteria which were chosen because it has been used in several studies, including the WHFS, $[14,15]$ and is straightforward to apply. These criteria include the presence of 2 major (e.g. radiological evidence of pulmonary edema) or 1 major and 2 minor (e.g. extremity oedema, exertional dyspnoea) criteria [13]. Subjects were excluded if they had hypotension (SBP $<95 \mathrm{mmHg}$ ) or cardiogenic shock, or if they were on haemodialysis.

\section{Data collection activities}

Both contributing studies collected the majority of their data from structured chart reviews. Data included patients' demographic characteristics, past medical history, physical exam findings, vital signs,

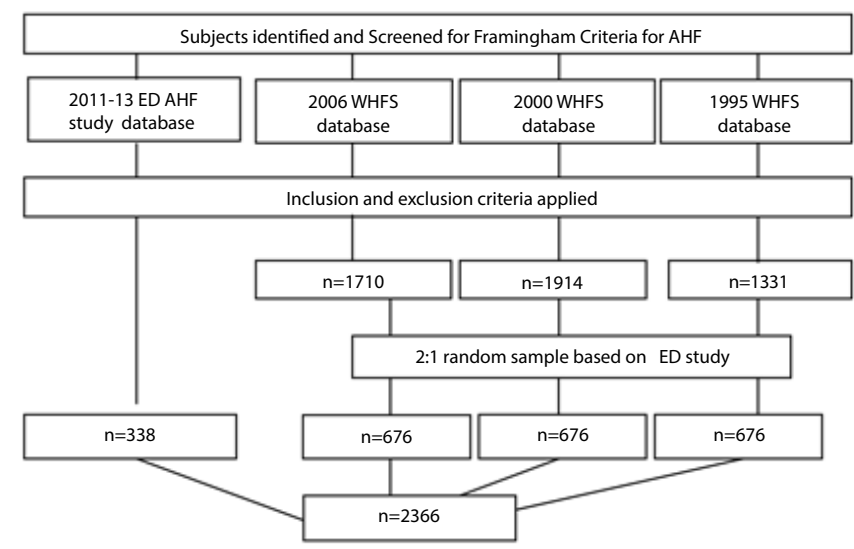

Figure 1: Diagram of subject enrolment.

home medications, clinical characteristics, admission status (e.g., ICU versus floor), and laboratory results. For the main variable of interest, SBP, we used the first available reading obtained after the patient arrived at the hospital ED. The chart review also obtained information on short-term clinical outcomes including inpatient length of stay (LOS), need for ICU admission, endotracheal intubation, complications, and ejection fraction findings.

\section{Data Analysis}

\section{Methods used to summarize the data}

A total of 338 subjects were available from the ED-based AHF study for analysis. To increase statistical power, we took a 2:1 random sample of subjects from each WHFS year (compared to the ED-based study) which resulted in a total of 676 patients in each WHFS cohort $(1995,2000,2006)$ (Figure 1). Differences in the demographic and clinical characteristics of patients in each of the four study groups were compared by chi-square tests for categorical and ANOVA for continuous variables (SAS, Cary, NC).

\section{Methods used to test the principal study hypothesis}

Our primary study endpoint was the percentage of patients presenting to the ED with AHF who had a SBP $>160 \mathrm{mmHg}$ during each of the years under study. Our hypothesis was that the frequency of AHF presentations accompanied by hypertension (SBP $>160 \mathrm{mmHg}$ ) had declined over the study interval. We used a SBP cutoff of $>160 \mathrm{mmHg}$ because it was the highest BP category defined in the OPTIMIZE-HF registry study [9]. In published guidelines it identifies patients with AHF who are in a low risk group, [16] and also because this group would be most likely to require acute treatment to lower their BP in the ED. For the univariate analyses, differences in the average SBP at the time of ED presentation according to study year were compared by chi-square tests of statistical significance. In addition, a multivariate logistic regression analysis was performed to examine whether study year was associated with a decrease in hypertensive AHF presentations over time at a SBP cut point of $160 \mathrm{mmHg}$. We set 1995 as the referent year and created a multiple logistic regression model by adding potential confounding variables to the analysis based on clinical relevance and a $\mathrm{p}$ value of $<0.20$ on univariate examination. These factors included age, sex, race, body mass index, hematocrit, outpatient medications, a history of hypertension, atrial fibrillation, diabetes mellitus, kidney disease, and hypercholesterolemia. We also considered additional confounding factors of prognostic importance that have been shown to be associated with mortality and other clinical outcomes in patients with AHF 
Citation: Darling CE, Sun JE, Goldberg J, Pang P, Baugh CW, et al. (2017) A Historical Perspective on Presentations of Hypertensive Acute Heart Failure. J Cardiovasc Dis Diagn 5: 275. doi: 10.4172/2329-9517.1000275

Page 3 of 6

$[17,18]$. Of note, EF findings were missing in $58 \%$ of the study sample and were not controlled for in the regression models. Similarly, B-type natriuretic peptide (BNP) levels were not available in the 1995 and 2000 study cohorts and were not included in our regression analyses.

\section{Results}

\section{Characteristics of the entire study population}

A total of 2,366 individuals admitted with AHF comprised our study population (Figure 1). The average age of this population was 77 years, $55 \%$ were female, $92 \%$ were white, and $75 \%$ had been previously diagnosed with HF. Between 1995 and 2011-13 there were several notable changes in the clinical characteristics of these patients. Compared to patients admitted in 1995, patients in the more recent study years of 2011-13 were significantly more likely to have a history of hypertension, atrial fibrillation, chronic kidney disease, and hyperlipidemia. These patients were also more likely to be taking ACEInhibitors and beta-blockers and have a shorter length of hospital stay, but were less likely to be taking digoxin than patients in 1995 (Table 1).

\section{Trends in the characteristics of patients with SBP $>160 \mathrm{mmHg}$}

Among patients with AHF and a SBP $>160 \mathrm{mmHg}$, the average age was 76 years, $61 \%$ were female, and $91 \%$ were white. Consistent with the findings in the entire study sample, between 1995 and 2011-13 the characteristics of patients with AHF and a presenting SBP $>160 \mathrm{mmHg}$ also changed. In more recent study years, AHF patients with systolic

\begin{tabular}{|c|c|c|c|c|c|}
\hline & \multicolumn{5}{|c|}{ Study Year } \\
\hline & 1995 & 2000 & 2006 & 2011-13 & $p$-value \\
\hline Demographics & $(n=676)$ & $(n=676)$ & $(n=676)$ & $(n=338)$ & \\
\hline Age (mean, yrs) & 76.7 & 76.9 & 77.6 & 74.8 & $<0.01$ \\
\hline Female (\%) & 57.9 & 56.7 & 56.4 & 41.2 & $<0.01$ \\
\hline White (\%) & 95.9 & 91.4 & 90.1 & 89.6 & $<0.01$ \\
\hline Body mass index $\left(\mathrm{kg} / \mathrm{m}^{2}\right)$ & 26.9 & 27.7 & 28.0 & 31.7 & $<0.01$ \\
\hline & Medical /S & urgical $\mathrm{Hi}$ & ory (\%) & & \\
\hline Hypertension & 64.4 & 68.1 & 82.7 & 89.4 & $<0.01$ \\
\hline Atrial fibrillation & 36.7 & 36.7 & 45.9 & 53.9 & $<0.01$ \\
\hline Diabetes mellitus & 39.5 & 38.2 & 37.9 & 52.7 & $<0.01$ \\
\hline Coronary heart disease & 56.7 & 58.4 & 58.1 & 58.3 & 0.6 \\
\hline $\begin{array}{l}\text { Chronic pulmonary } \\
\text { disease }\end{array}$ & 38.0 & 34.6 & 40.1 & 35.8 & 0.6 \\
\hline Stroke & 15.8 & 16.4 & 14.1 & 18.6 & 0.9 \\
\hline Chronic kidney disease & 20.6 & 22.0 & 36.5 & 46.5 & $<0.01$ \\
\hline Heart failure & 75.3 & 73.3 & 79.1 & 71.9 & 0.6 \\
\hline Hypercholesterolemia & 14.4 & 24.9 & 53.3 & 73.7 & $<0.01$ \\
\hline Mean EF (\%) & 40.7 & 43.2 & 47.3 & 44.4 & $0.51^{*}$ \\
\hline & Home I & ledication & (\%) & & \\
\hline ACE-Inhibitors & 39.1 & 36.1 & 39.6 & 55.9 & $<0.01$ \\
\hline Beta Mockers & 21.3 & 40.4 & 64.4 & 74.9 & $<0.01$ \\
\hline ACE-I + Beta Stockers & 7.0 & 17.5 & 28.7 & 44.4 & $<0.01$ \\
\hline $\begin{array}{l}\text { Calcium Channel } \\
\text { Blockers }\end{array}$ & 33.6 & 24.0 & 23.8 & 26.3 & $<0.01$ \\
\hline Diuretics (any) & 70.4 & 66.7 & 72.0 & 77.5 & $<0.01$ \\
\hline Digoxin & 39.9 & 33.1 & 18.1 & 18.3 & $<0.01$ \\
\hline & Labor & tory fin & & & \\
\hline Creatinine & 1.5 & 1.5 & 1.5 & 1.6 & 0.5 \\
\hline Hematocrit (\%) & 37.2 & 36.2 & 35.3 & 35.1 & $<0.01$ \\
\hline
\end{tabular}

\# - missing data in $>50 \%$ of patients, ACE-I= angiotensin converting enzymeinhibitor, Creatinine (mg/dl)

Table 1: Selected demographic and clinical characteristics of the entire study sample.

\begin{tabular}{|c|c|c|c|c|c|}
\hline & \multicolumn{5}{|c|}{ Study Year } \\
\hline & 1995 & 2000 & 2006 & 2011-13 & \multirow{2}{*}{ p-value } \\
\hline Demographics & $(n=227)$ & $(n=189)$ & $(n=143)$ & $(n=66)$ & \\
\hline Age (mean, yrs) & 76.8 & 76.4 & 76.7 & 73.5 & 0.25 \\
\hline Female (\%) & 66.5 & 61.4 & 53.9 & 54.6 & $<0.01$ \\
\hline White (\%) & 96 & 87.3 & 89.5 & 90 & 0.09 \\
\hline Body mass index $\left(\mathrm{kg} / \mathrm{m}^{2}\right)$ & 27.9 & 28.6 & 28.3 & 33.4 & $<0.01$ \\
\hline \multicolumn{6}{|c|}{ Medical/Surgical History (\%) } \\
\hline Hypertension & 75.3 & 77.8 & 88.1 & 93.9 & $<0.01$ \\
\hline Atrial fibrillation & 32.6 & 29.6 & 30.8 & 45.5 & 0.23 \\
\hline Diabetes mellitus & 44.9 & 42.3 & 39.9 & 50 & 0.96 \\
\hline Coronary heart disease & 55.1 & 57.1 & 49 & 54.6 & 0.36 \\
\hline $\begin{array}{c}\text { Chronic obstructive pulmonary } \\
\text { disease }\end{array}$ & 35.2 & 31.2 & 34.3 & 25.8 & 0.36 \\
\hline Stroke & 18.1 & 12.1 & 16.8 & 25.8 & 0.27 \\
\hline Chronic kidney disease & 19.8 & 22.8 & 37.8 & 42.4 & $<0.01$ \\
\hline Heart failure & 72.3 & 66.1 & 72 & 60.6 & 0.35 \\
\hline Hyperlipidemia & 19.4 & 25.4 & 48.3 & 74.2 & $<0.01$ \\
\hline \multicolumn{6}{|c|}{ Home Medications (\%) } \\
\hline ACE-Inhibitors & 36.1 & 34.4 & 39.9 & 62.1 & $<0.01$ \\
\hline Beta Blockers & 26.9 & 48.2 & 62.9 & 77.3 & $<0.01$ \\
\hline ACE-I + Beta Blockers & 7.5 & 21.1 & 29.4 & 50 & $<0.01$ \\
\hline Digoxin & 36.6 & 26.5 & 14 & 7.6 & $<0.01$ \\
\hline Diuretics (any) & 64.8 & 56.6 & 60.1 & 69.7 & 0.81 \\
\hline \multicolumn{6}{|c|}{ Laboratory findings } \\
\hline Creatinine, mean (mg/dl) & 1.46 & 1.5 & 1.5 & 1.55 & 0.8 \\
\hline Hematocrit mean (\%) & 38.1 & 36.9 & 36.2 & 35.9 & 0.03 \\
\hline
\end{tabular}

Table 2: Selected demographic and clinical characteristics of patients of patients with a SBP $>160 \mathrm{mmHg}$ on presentation.

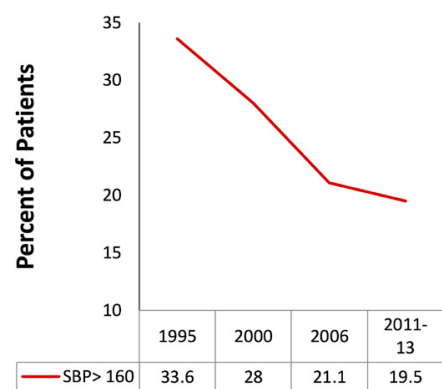

Figure 2: Percent of patients with AHF presenting with systolic blood pressure $>160 \mathrm{mHg}$ over time.

hypertension on presentation were less likely to be women, more likely to have a shorter LOS and a lower mean SBP on admission, and fewer were prescribed digoxin (Table 2).

\section{Mean SBP and the frequency of elevated SBP over time}

The SBP at presentation averaged $151 \mathrm{mmHg}$ in 1995 and decreased over time to $140 \mathrm{mmHg}$ by $2011-2013$ ( $\mathrm{p}<0.01$ ). In $1995,33.6 \%$ of all AHF patients had a SBP $>160 \mathrm{mmHg}$ which decreased to $19.5 \%$ by 2011-2013 $(\mathrm{p}<0.01)$ (Figure 2). 45\% of patients had ejection fraction (EF) measured during their index inpatient admission. For the subset of patients with EF measurements, SBP trends were analyzed by either preserved $(\geq 50 \%)$ or reduced $(\leq 40 \%)$ EF. In patients with reduced $\mathrm{EF}$, average SBP declined from $152 \mathrm{mmHg}$ in 1995 to $138 \mathrm{mmHg}$ in 2011-13 ( $p=0.03$ ). However, for those patients with preserved EF SBP declines were much less significant and averaged $156 \mathrm{mmHg}$ in 1995 and $149 \mathrm{mmHg}$ in 2011-13. 


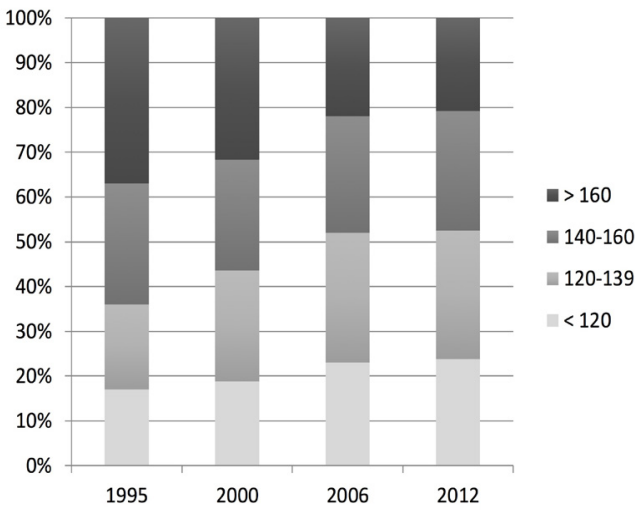

Figure 3: Distribution of systolic blood pressure $(\mathrm{mmHg})$ by study year.

\begin{tabular}{|c|c|c|}
\hline Year & Crude OR & Adjusted OR \\
\hline $1995^{\circ}$ & 1 & 1 \\
\hline \multirow{2}{*}{2000} & 0.7 & 0.94 \\
\hline \multirow{2}{*}{2006} & $(0.54-0.90)^{\$}$ & $(0.67-1.31)$ \\
\hline \multirow{2}{*}{$2011-13$} & 0.41 & 0.64 \\
\hline & $(0.30-0.55)$ & $(0.42-0.96)$ \\
\hline & 0.35 & 0.46 \\
\hline
\end{tabular}

* adjusted for the following variables: age, sex, race, body mass index, hypertension, atrial fibrillation, diabetes mellitus, kidney disease,

hypercholesterolemia, outpatient medication (ace-inhibitors, beta-blockers, diuretics, digoxin, calcium channel blockers, hematocrit; ${ }^{0} 1995$ is the referent year; $\$ 95 \% \mathrm{Cl}$

Table 3: Crude and adjusted odds ratios for patients presenting with a SBP $>160$ $\mathrm{mmHg}$.

The distribution of SBP across four BP strata has also changed over time. Patients hospitalized with AHF during more recent years (after 2006) demonstrated a more balanced and even distribution of SBP than earlier years where a greater percentage of patients fell into the higher (>140 $\mathrm{mmHg}$ and $>160 \mathrm{mmHg}$ ) SBP strata (Figure 3).

When compared to patients hospitalized in 1995 (referent year), the crude odds ratios demonstrated that there was a significantly lower odds of patients with AHF presenting with SBP $>160 \mathrm{mmHg}$ in 2000, 2006, and 2011-2013. After adjustment for several demographic, medical history, and clinical factors, only the 2006 and 2011-13 cohorts were found to have a significantly reduced odds of presenting with a SBP $>160 \mathrm{mmHg}$ (Table 3).

\section{Discussion}

In the present study we compared the frequency of patients with AHF presenting to the ED with SBP greater than $160 \mathrm{mmHg}$ in four cross-sectional study cohorts between 1995 and 2011-13. The rationale for studying presenting SBP at presentation for patients with AHF is to try to understand if there was a change in the epidemiology of these patients over time and because of expert recommendations to use SBP as a guide to early diagnostic and treatment approaches. Our results demonstrate that there was a significant decrease over our study time period in the proportion of AHF patients who present with SBP $>160$ $\mathrm{mmHg}$, as well as a similar decrease in the mean SBP over greater than 15 years beginning in 1995 .

Despite a decrease in the proportion of patients with an SBP $>160 \mathrm{mmHg}$ over time, the overall mean SBP results we observed are consistent with other observational studies of patients with AHF conducted in the same time period. The acute decompensated heart failure national registry (ADHERE) collected data on patients with AHF who were admitted to 263 medical centers from all regions of the United States between 2001 and 2006. In ADHERE, patients admitted between 2001 and 2003 were found to have a mean initial SBP of $144 \mathrm{mmHg}$ which falls between our mean SBP's for 2000 and 2006 which were $147 \mathrm{mmHg}$ and $141 \mathrm{mmHg}$ respectively [19]. Similarly, the organized program to initiate lifesaving treatment in hospitalized patients with heart failure (OPTIMIZE-HF) national registry found that the average SBP of patients admitted with AHF in 2003 and 2004 was $143 \mathrm{mmHg}$. Although we found a decline in the initial SBP of patients with AHF over our study time-period it is important to note that most still had normal to elevated SBP at the time of hospital arrival. Our results also suggest that patients with preserved ejection fraction were the least affected by this overall trend of reduced initial SBP over time.

The importance of SBP in providing a framework for clinicians to clinically categorize and better manage patients with AHF has been discussed in several studies $[5,7,8]$. The OPTIMIZE-HF registry of nearly 50,000 patients found that patients with higher SBP's were more likely to be female, African American, and to have heart failure with a preserved ejection fraction [9]. OPTIMIZE-HF also documented the relationship between initial SBP and short-term (inpatient) and post discharge prognosis, where patients having higher SBPs $(>140$ $\mathrm{mmHg}$ ) were found to have a decreased mortality compared with patients in lower SBP strata [9]. Lastly, an American Heart Association working group focusing on the diagnosis and treatment of AHF in the ED setting has advocated an approach whereby SBP is central to early risk stratification with patients having a SBP $>160 \mathrm{mmHg}$ falling into the lowest risk prognostic group [16]. Moreover, prior literature has characterized patients with AHF and significantly elevated SBP as having "vascular failure" rather than "cardiac failure". This distinction is helpful to clinicians as they attempt to distinguish between patients with different treatment needs $[8,20]$. The vascular failure patient typically does not have significant total body fluid overload and, as a result, early treatment focuses on afterload reduction rather than intensive diuresis $[8,20]$.

It is unclear why the proportion of patients with AHF and hypertension underwent the historical decline we observed. However, our data demonstrates that many demographic and clinical features of these patients have also changed during the last few decades. Since 1995, patients enrolled in studies of AHF in our geographic area have become older, have a greater comorbid disease burden, but have an improved post discharge prognosis [21]. Similarly, other studies have documented improvement in the long-term survival of patients discharged from the hospital after AHF treatment $[22,23]$. A large North American study of more than 55 million Medicare patients discharged with a diagnosis of AHF found that admission rates for AHF declined significantly between 1998 and 2008, perhaps reflecting improved management of these patients [3]. Overall these changes may partly explain our results.

However, other factors may also explain our findings. First, the outpatient management of heart failure changed significantly while our data was collected [24,25]. There is also increased recognition that outpatient hypertension management in HF patients can decrease the incidence rate of AHF presentations [26]. In our sample there was a steady increase in the use of ACE-Inhibitors and beta-blockers in isolation and in combination over time. Others have documented similar increases in the use of these medications over time [27]. It is unclear how the outpatient management of patients with $\mathrm{HF}$ influences the SBP when a patient presents with AHF, but it may, in part, explain the trends we observed. 
Lastly, the management of hypertension has changed significantly with new definitions of hypertension and targets for therapy proposed with each Joint National Committee on the Detection, Evaluation, and Treatment of High Blood Pressure (JNC) update since 1977 [28]. In our study, we found a significant increase in the proportion of patients with pre-existing hypertension over time which likely reflects the increased comorbid disease burden in these patients, the changing definitions of hypertension, and the heightened focus on this disease. Since hypertension is an important cause of heart failure, and blood pressure control has improved over time, these changing trends may have influenced our results $[28,29]$.

\section{Study Strengths and Limitations}

The major strength of this study lies in its ability to combine detailed current and historical data in order to better understand how the presentation of patients with AHF may have changed over time. However, there are several limitations that should be considered when interpreting our findings. First, the WHFS data were compared to an ED-based AHF study and there were some differences in how the patients were identified. Such heterogeneity could introduce a potential selection bias. However, the significant trend in reduction of hypertensive presentations in patients with AHF was also observed when only considering the most recent WHFS cohort (2006) (excluding ED-based 2011-13 data), reinforcing the likelihood of our observed trends being real. Secondly there was no standard method by which the patient's initial SBP was measured although this limitation would have been consistent across all study years. Third, ejection fraction (EF) is an important variable that is related to presenting SBP, but data on EF were missing in a large proportion of subjects and was not included in our regression models. Similarly, BNP was not obtained in the first 2 patient cohorts due to it not being used at all the study sites. Changes in prehospital treatment may also have influenced the initial presenting SBP's, but the proportion of patients with AHF transported by ambulance has not significantly changed over time (data not shown). Patient with a SBP $<95 \mathrm{mmHg}$ was excluded from both studies due to the initial goals of the ED-based research protocol. This could result in the mean SBP's we report to be somewhat higher than values reported in other studies. This study was also conducted in a single region in the USA, which may limit the generalizability of our findings to other geographic areas where the racial, socio-demographic, and clinical characteristics of patients differ.

\section{Conclusion}

Our study demonstrates that the proportion of patients with AHF accompanied by a SBP $>160 \mathrm{mmHg}$ at the time of presentation to the hospital declined significantly between 1995 and the late 2000s. In addition, the mean SBP at presentation also declined over the nearly 2 decade period covered under this study. Why these changes have occurred is unknown, but they are likely a result of the changes that have occurred in the outpatient management of both heart failure and hypertension over the last 15-20 years. It also highlights the need for ongoing surveillance studies of patients with AHF as the risk of HF increases in prevalence with the aging of the population.

\section{Acknowledgements}

This work was supported by grants from the National Institute of Health through the National Heart, Lung, and Blood Institute [R37 HL69874 and K23HL101991].

\section{Conflicts of Interest:}

None declared.

\section{References}

1. McMurray JJ, Petrie MC, Murdoch DR, Davie AP (1998) Clinical epidemiology of heart failure: Ppublic and private health burden. European heart journal 19 9-16.

2. Collins SP, Lindsell CJ, Storrow AB, Fermann GJ, Levy PD, et al. (2012) Early changes in clinical characteristics after emergency department therapy for acute heart failure syndromes: Identifying patients who do not respond to standard therapy. Heart Fail Rev 17: 387-394.

3. Chen J, Normand SL, Wang Y, Krumholz HM (2011) National and regional trends in heart failure hospitalization and mortality rates for Medicare beneficiaries, 1998-2008. JAMA 306: 1669.

4. Benjamin EJ, Blaha MJ, Chiuve SE, Cushman M, Das SR, et al. (2017) Heart disease and stroke statistics-2017 update: A report from the American Heart Association. Circulation 135: e146-603.

5. Weintraub NL, Collins SP, Pang PS, Levy PD, Anderson AS, et al. (2010) Acute heart failure syndromes: Emergency department presentation, treatment, and disposition-Current approaches and future aims: A scientific statement from the American Heart Association. Circulation 122: 1975-1996.

6. Collins S, Storrow AB, Kirk JD, Pang PS, Diercks DB, et al. (2008) Beyond pulmonary edema: Diagnostic, risk stratification, and treatment challenges of acute heart failure management in the emergency department. Ann Emerg Med 51: 45-57.

7. Gheorghiade M, Pang PS (2009) Acute heart failure syndromes. J Am Coll Cardiol 53: 557-573.

8. Gheorghiade M, De Luca L, Fonarow GC, Filippatos G, Metra M, et al. (2005) Pathophysiologic targets in the early phase of acute heart failure syndromes. Am J Cardiol 96: 11G-7G.

9. Gheorghiade M, Abraham WT, Albert NM, Greenberg BH, O'Connor CM et al. (2006) Systolic blood pressure at admission, clinical characteristics, and outcomes in patients hospitalized with acute heart failure. JAMA 296 2217-2226.

10. Cotter G, Metra M, Milo-Cotter O, Dittrich HC, Gheorghiade M (2008) Fluid overload in acute heart failure--re-distribution and other mechanisms beyond fluid accumulation. Eur J Heart Fail 10: 165-169.

11. Joffe SW, Webster K, McManus DD, Kiernan MS, Lessard D, et al. (2013) Improved survival after heart failure: A community-based perspective. J Am Heart Assoc 2: e000053.

12. Goldberg RJ, Darling C, Joseph B, Saczynski J, Chinali M, et al. (2009) Epidemiology of decompensated heart failure in a single community in the northeastern United States. Am J Cardiol 104: 377-382.

13. McKee PA, Castelli WP, McNamara PM, Kannel WB (1971) The natura history of congestive heart failure: The Framingham study. N Engl J Med 285: $1441-1446$

14. Bhatia RS, Tu JV, Lee DS, Austin PC, Fang J, et al. (2006) Outcome of heart failure with preserved ejection fraction in a population-based study. $\mathrm{N}$ Engl J Med 355: 260-269.

15. Ho KK, Anderson KM, Kannel WB, Grossman W, Levy D (1993) Survival after the onset of congestive heart failure in Framingham heart study subjects. Circulation 88: 107-115.

16. Peacock WF, Braunwald E, Abraham W, Albert N, Burnett J, et al. (2010) National heart, lung, and blood institute working group on emergency department management of acute heart failure: Research challenges and opportunities. J Am Coll Cardiol 5: 343-351.

17. Yancy CW, Lopatin M, Stevenson LW, De Marco T, Fonarow GC (2006) Clinical presentation, management, and in-hospital outcomes of patients admitted with acute decompensated heart failure with preserved systolic function: A report from the acute decompensated heart failure national registry (ADHERE) Database. J Am Coll Cardiol 47: 76-84.

18. Peacock WF, Fonarow GC, Emerman CL, Mills RM, Wynne J (2007) Impact of early initiation of intravenous therapy for acute decompensated heart failure on outcomes in ADHERE. Cardiology 107: 44-51.

19. Fonarow GC, Adams KF Jr., Abraham WT, Yancy CW, Boscardin WJ (2011) Risk stratification for in-hospital mortality in acutely decompensated heart failure: Classification and regression tree analysis. JAMA 293: 572-580.

20. Gheorghiade M, Braunwald E (2011) A proposed model for initial assessment and management of acute heart failure syndromes. JAMA 305: 1702-1703.

21. Park D, McManus D, Darling C, Goldberg JH, Gore JM, et al. (2012) Recent 
Citation: Darling CE, Sun JE, Goldberg J, Pang P, Baugh CW, et al. (2017) A Historical Perspective on Presentations of Hypertensive Acute Heart Failure. J Cardiovasc Dis Diagn 5: 275. doi: 10.4172/2329-9517.1000275

trends in the characteristics and prognosis of patients hospitalized with acute heart failure. Clin Epidemiol 3: 295-303.

22. Shahar E, Lee $S$ (2007) Historical trends in survival of hospitalized heart failure patients: 2000 versus 1995. BMC Cardiovasc Disord 7: 2

23. Roger VL, Weston SA, Redfield MM, Hellermann-Homan JP, Killian J, et al. (2004) Trends in heart failure incidence and survival in a community-based population. JAMA 292: 344-350.

24. Yancy CW, Jessup M, Bozkurt B, Butler J, Casey DE Jr, et al. (2017) 2017 ACC/AHA/HFSA Focused update of the 2013 ACCF/AHA guideline for the management of heart failure: A report of the American College of Cardiology/ American Heart association task force on clinical practice guidelines and the heart Failure Society of America. Circulation.

25. Williams JF, Bristow MR, Fowler MB, Francis GS, Garson A, et al. (1995) Guidelines for the evaluation and management of heart failure. Circulation 92:
2764-2784.

26. Kostis JB, Davis BR, Cutler J, Grimm RH, Jr., Berge KG, et al. (1997) Prevention of heart failure by antihypertensive drug treatment in older persons with isolated systolic hypertension. SHEP Cooperative Research Group. JAMA 278: 212-216.

27. Smith NL, Chan JD, Rea TD, Wiggins KL, Gottdiener JS, et al. (2004) Time trends in the use of beta-blockers and other pharmacotherapies in older adults with congestive heart failure. Am Heart J 148: 710-717.

28. James PA, Oparil S, Carter BL, Cushman WC, Dennison-Himmelfarb C, et al. (2014) 2014 evidence-based guideline for the management of high blood pressure in adults: Report from the panel members appointed to the Eighth Joint National Committee (JNC 8). JAMA 311: 507-520.

29. Wang TJ, Vasan RS (2005) Epidemiology of uncontrolled hypertension in the United States. Circulation 112: 1651-1662. 